\title{
Crossed acoustic response combined with visual and somatosensory evoked responses in the diagnosis of multiple sclerosis
}

\author{
R. E. CLIFF OR D-J O NES, G. P. CLARKE, A ND P. M A Y LES \\ From the Departments of Neurology and Physics, Guy's Hospital, London
}

SUMMARY The crossed acoustic response, (CAR), a recently introduced test of brainstem function, has been studied in 66 patients with multiple sclerosis and 53 control subjects, and compared with conventional visual and somatosensory evoked responses (VER, SER). A latency abnormality was found in the CAR in $73 \%$ of patients, in the VER in $63 \%$, and in the SER in $37 \%$. Abnormalities have been related to the presence or absence of clinically detectable signs. All three responses detected subclinical lesions by showing abnormality in a proportion of multiple sclerosis patients who had no corresponding abnormal clinical signs (CAR 69\%, VER $42 \%$, SER $29 \%$ ). The best diagnostic combination of responses was VER and CAR. Ninety per cent of patients had at least one of these two responses abnormal.

The diagnosis of multiple sclerosis depends on demonstrating objective evidence of multiple lesions within the central nervous system white matter of a patient with a suitable history and no alternative explanation (McDonald and Halliday, 1977). The electrophysiological demonstration of abnormalities in clinically normal parts of the nervous system has been shown to be of assistance in early diagnosis (Halliday et al., 1973; Asselman et al., 1975; Starr and Achor, 1975; Humphries et al., 1976a; Robinson and Rudge 1975, 1977; Small et al., 1977).

We have used the crossed acoustic response (CAR) as an indicator of brainstem function and have compared it with visual and somatosensory evoked responses (VER, SER). The results obtained with the CAR compare favourably with other tests of brainstem function (Kimura, 1975; Robinson and Rudge, 1975, 1977; Mastaglia et al., 1977), and therefore the CAR with the VER and SER provide a useful combination of responses for detecting subclinical demyelination.

\section{Patients and methods}

Sixty-six patients of mean age 44 years (standard

Address for reprint requests: Dr R. E. Clifford-Jones, Department of Clinical Neurology, The National Hospital, Queen Square, London WC1N 3BG.

Accepted 20 February 1979 deviation 12.8 years) attending Guy's Hospital were classified according to McAlpine's criteria for the diagnosis of multiple sclerosis (McAlpine et al., 1972). Twenty-one patients had definite, 14 probable, and 31 possible multiple sclerosis. The patients were compared with 53 control subjects with no known neurological disease, mean age 33 years (SD 11.6). However, all three tests were not done on all normal subjects.

A full general and detailed neurological assessment was made at or near the time when the patient's responses were measured. The presence of abnormality within the brainstem, visual, and somatosensory systems was noted. Forty per cent of patients had abnormal signs in the visual system, $23 \%$ in the brainstem, $25 \%$ in the sorizatosensory system, and $26 \%$ in at least two of the three systems.

Most patients (47 out of 66) had all three responses measured and as far as possible they were done on the same or consecutive days. The crossed acoustic response (CAR) (Yoshie et al., 1969; Thornton, 1975; Humphries et al., 1976a, b) was the myogenic response from the postauricular muscles to monaural clicks as described by Douek et al. (1973). This is a bilateral response which crosses the brainstem and involves a pathway between one ear and the postauricular muscles of both sides. The latencies of the responses in the ipsilateral and contralateral muscles to stimula- 
tion of one ear were measured and compared with the latencies obtained from stimulation of the other ear (Figure). The greatest latency difference between any two of these four responses was then calculated. It was possible to obtain satisfactory responses in virtually all subjects (62 out of 66 patients, 52 out of 53 control subjects) although in some it was necessary to employ methods (such as resisted neck flexion) which increased the tone in the postauricular muscles. Despite considerable variation in amplitude the maximum latency difference of the normal response was remarkably constant (see Results).

The visual evoked response (VER) was the midline occipital response to pattern reversal recorded by the method described by Halliday et al. (1973) with some minor modifications. The somatosensory response (SER) was the contralateral parietal response to percutaneous electric shocks to the median nerve at the wrist as described by Namerow (1968).

\section{Results}

The means and standard deviations (SD) of the latencies of the three responses are shown in Table 1. A patient's response was classified as abnormal if its latency exceeded the normal mean $+3 \mathrm{SD}$. On this criterion the percentages of $\mathrm{ab}$ - normal responses in the multiple sclerosis groups $Z$ are shown in Table 2 . Table 3 relates the abnormal responses for all multiple sclerosis patients to theo

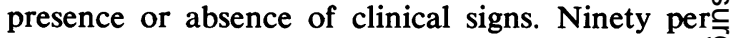
cent of 41 patients had either the VER or CAR or both abnormal, which is greater than thew percentages detected by the combinations VER? and SER (71\%) and CAR and SER (80\%).

Table 1 Means and standard deviations of latencies in ms. Mean (SD)

\begin{tabular}{lrrrrr}
\hline & Normal & Possible MS & Probable MS Definite MS \\
\hline VER & - ve $96.0(5.3)$ & $105.1(8.8)$ & $119.1(19.5)$ & $115.5(18.7)$ \\
& +ve $120.8(5.6)$ & $131.8(12.8)$ & $146.3(19.8)$ & $147.8(23.9)$ \\
CAR* & $0.6(0.3)$ & $2.5(2.1)$ & $2.8(1.4)$ & $3.8(2.9)$ \\
SER & $32.7(4.0)$ & $40.0(8.9)$ & $44.6(17.0)$ & $41.5(11.5)$ \\
\hline
\end{tabular}

*Figures represent the maximum latency difference (see text).

Table 2 Abnormal responses in multiple sclerosis patients

\begin{tabular}{lcccc}
\hline & Definite $M S$ & Probable $M S$ & Possible $M S$ & Tota \\
\hline VER & $25 / 31(81 \%)$ & $8 / 11(73 \%)$ & $6 / 20(30 \%)$ & $63 \%$ \\
CAR & $23 / 31(74 \%)$ & $11 / 13(84 \%)$ & $13 / 20(65 \%)$ & $73 \%$ \\
SER & $8 / 23(35 \%)$ & $5 / 12(41 \%)$ & $6 / 16(37.5 \%)$ & $37 \%$ \\
\hline
\end{tabular}

70
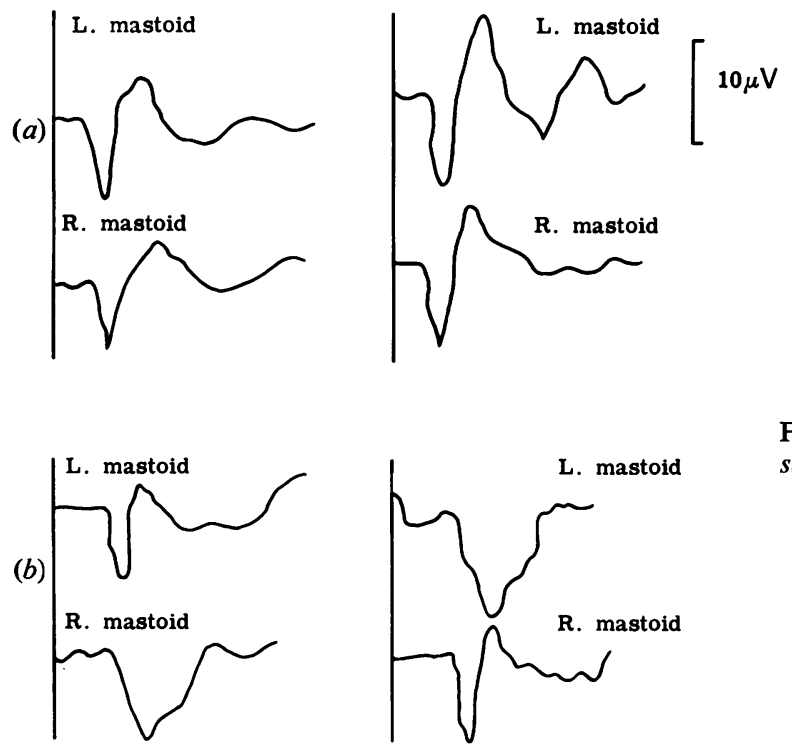

Left ear stimulation

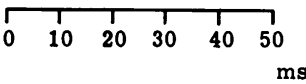

Right ear stimulation

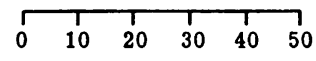

Figure Crossed acoustic response (a) in a normal subject and $(b)$ in a patient with multiple sclerosis. 
Table 3 Abnormal responses in relation to clinical signs

\begin{tabular}{llr}
\hline & Without signs & With signs \\
\hline VER & $14 / 33(42 \%)$ & $25 / 29(86 \%)$ \\
CAR & $34 / 49(69 \%)$ & $13 / 15(87 \%)$ \\
SER & $11 / 38(29 \%)$ & $8 / 13(62 \%)$ \\
\hline
\end{tabular}

\section{Discussion}

The abnormal CARs which we have found in multiple sclerosis patients extend the findings described in a previous small series (Humphries et al., 1976a). This test, which is easy and quick to do, yields a percentage of abnormality ( $73 \%$ of all multiple sclerosis patients) which is higher than other tests of brainstem function (Kimura, 1975; Robinson and Rudge, 1975, 1977; Mastaglia et al., 1977).

Our VER and SER results confirm the presence of delayed responses found in other series. Reports on VER are by Halliday et al. (1973), Asselman et al. (1975), Feinsod et al. (1975), Mastaglia et al. (1976), Regan et al. (1976), and on SER by Halliday and Wakefield (1963), Giblin (1964), Baker et al. (1968), Namerow (1968), and Small et al. (1977). The proportion of our patients with delayed VERs $(63 \%)$ is similar to several other series but lower than the $92 \%$ reported by Halliday. As Asselman et al. (1975) discuss, population structure, stimulus characteristics, and criteria for the definition of abnormality all influence the percentages of abnormal responses found. As neither a different pattern size $\left(54^{\prime}\right)$ nor less stringent abnormality criteria (mean $+2.5 \mathrm{SD}$ ) give very different results when applied to our data the difference from Halliday is probably the result of patient selection. The number of our patients with delayed SER $(37 \%)$ is lower than in previous series. This was mainly because of the strict criterion we have used to define abnormality. We have defined abnormality solely in terms of a latency exceeding the mean $+3 \mathrm{SD}$. Other workers (Namerow, 1968) have used $2 \mathrm{SD}$, and on this basis our results would be comparable. The percentages of abnormal CARs and VERs in patients who have the corresponding clinical signs present are equal and just significantly higher $(P<0.1)$ than the percentage of abnormal SERs (Table 3). However, if no corresponding clinical signs are detected the percentage of abnormal CARs $(69 \%)$ is significantly greater than that of either VER or SER $(P<0.05)$. This is an index of detection of subclinical lesions and it suggests either that the CAR is the most sensitive of the three tests or that definite brainstem signs are difficult to detect clinically. As the VER and CAR seem equally sensitive in detecting clinically apparent lesions, the second explanation seems the more probable.

By measuring a combination of responses the detection of abnormality in multiple sclerosis patients is increased. The best diagnostic combination of responses was found to be CAR and VER. Ninety per cent of patients had at least one of these two responses delayed. This compares favourably with other studies using a combination of responses (Mastaglia et al., 1976, 1977; Deltenre et al., 1978).

The real advantage of evoked responses in the diagnosis of multiple sclerosis is their ability to detect abnormalities in patients with few abnormal signs and thus to establish the existence of multiple lesions. In practice, the most appropriate responses to measure in a given patient are those in systems in which the patient has no clinical deficit. The particular value of the CAR is its high subclinical pick-up of lesions in a system where clinical signs may be difficult to detect.

We conclude that the recording of the CAR is a valuable test of brainstem function and with the VER provides a particularly useful combination of evoked responses for the detection of subclinical demyelination.

\section{References}

Asselman, P., Chadwick, D. W., and Marsden, C. D. (1975). Visual evoked responses in the diagnosis and management of patients suspected of multiple sclerosis. Brain, 98, 261-282.

Baker, J. B., Larson, S. J., Sances, A., and White, P. T. (1968). Evoked potentials as an aid to the diagnosis of multiple sclerosis. Neurology (Minneapolis), 18, 286.

Deltenre, P., Van Nechel, C., and Ketelaer, P. (1978). Evoked potentials in the early diagnosis of multiple sclerosis. Lancet, 1, 1040.

Douek, E., Gibson, W., and Humphries, K. (1973). The crossed acoustic response. Journal of Laryngology and Otology, 87, 711-726.

Feinsod, M., and Hoyt, W. F. (1975). Subclinical optic neuropathy in multiple sclerosis. Journal of Neurology, Neurosurgery, and Psychiatry, 38, 11091114.

Giblin, D. R. (1964). Somatosensory evoked potential in healthy subjects and in patients with lesions of the nervous system. Annals of the New York Academy of Sciences, 112, 93-142.

Halliday, A. M., and Wakefield, G. S. (1963). Cerebral evoked potentials in patients with dissociated sensory loss. Journal of Neurology, Neurosurgery, and Psychiatry, 26, 211-219. 
Halliday, A. M., McDonald, W. I., and Mushin, J. (1973). Visual evoked response in diagnosis of multiple sclerosis. British Medical Journal, 4, 661664.

Humphries, K. N., Ashcroft, P. N. C., Douek, E. E., and Clarke, G. P. (1976a). Delayed auditory evoked potentials in brainstem lesions. IERE Conference Proceedings, 34, 311-318.

Humphries, K. N., Gibson, W., and Douek, E. E. (1976b). Objective methods of hearing assessment: a system for recording the crossed acoustic response. Medical and Biological Engineering, 14, 1-8.

Kimura, J. (1975). Electrically elicited blink reflex in diagnosis of multiple sclerosis. Brain, 98, 413-425.

Mastaglia, F. L., Black, J. L., and Collins, D. W. K. (1976). Visual and spinal evoked responses in diagnosis of multiple sclerosis. British Medical Journal, $2,732$.

Mastaglia, F. L., Black, J. L., Cala, L. A., and Collins, D. W. K. (1977). Evoked potentials, saccadic velocities and computerised tomography in diagnosis of multiple sclerosis. British Medical Journal, 1, 1315-1317.

McAlpine, D., Lumsden, C. E., and Acheson, E. D. (1972). Multiple Sclerosis: A Reappraisal. Churchill Livingstone: Edinburgh.

McDonald, W. I., and Halliday, A. M. (1977). Diagnosis and classification of multiple sclerosis. British Medical Bulletin, 33, 4-8.
Namerow, N. S. (1968). Somatosensory evoked 으 responses in multiple sclerosis patients with varying sensory loss. Neurology (Minneapolis), 18, 11971204.

Regan, D., Milner, B. A., and Heron, J. R. (1976). Delayed visual perception and delayed visual evoked potentials in the spinal form of multiple sclerosis and in retrobulbar neuritis. Brain, 99, $\Omega$ 43-66.

Robinson, K., and Rudge, P. (1975). Auditory evoked $\stackrel{\overrightarrow{2}}{\overrightarrow{2}}$ responses in multiple sclerosis. Lancet, 1, 11641166.

Robinson, K., and Rudge, P. (1977). Abnormalities of the auditory evoked potentials in patients with multiple sclerosis. Brain, 100, 19-40.

Small, D. G., Beauchamp, M., and Matthews, W. B. (1977). Spinal evoked potentials in multiple sclerosis. Electroencephalography and Clinical Neurophysiology, 42, 141.

Starr, A., and Achor, L. J. (1975). Auditory brainstem responses in neurological disease. Archives of Neurology (Chicago), 32, 761-768.

Thornton, A. R. D. (1975). The use of post-auricular muscle responses. Journal of Laryngology and Otology, 89, 997-1010.

Yoshie, N., and Okudaira, T. (1969). Myogenic evoked potential responses to clicks in man. Act Oto-Laryngologica, Supplement 252, 89-103. 